\title{
Red light-induced citrus fruit colouration is attributable to increased carotenoid metabolism regulated by the NAC transcription factor FcrNAC22
}

\author{
Jinli Gong ${ }^{1}$, Yunliu Zeng ${ }^{1}$, Qiunan Meng ${ }^{1}$, Yajie Guan ${ }^{1}$, Chengyang Li $^{1}$, Hongbin Yang ${ }^{1}$, \\ Yingzi Zhang ${ }^{1}$, Charles Ampomah-Dwamena ${ }^{2}$, Ping Liu ${ }^{3}$, Chuanwu Chen ${ }^{3}$, Xiuxin Deng ${ }^{1}$, \\ Yunjiang Cheng ${ }^{1}$, and Pengwei Wang ${ }^{1}$ \\ ${ }^{1}$ Huazhong Agricultural University \\ ${ }^{2}$ The New Zealand Institute for Plant and Food Research Ltd (PFR), Private Bag 92169 \\ ${ }^{3}$ Guangxi Academy of Specialty Crops
}

November 4, 2020

\begin{abstract}
Carotenoids play vital roles in the colouration of plant tissues and organs, particularly fruits; however, the regulation of carotenoid metabolism in fruits during ripening is largely unknown. Here, we show that red light (RL) promotes fruit colouration by inducing accelerated degreening and carotenoid accumulation in kumquat fruits. Transcriptome profiling reveals that a NAC family transcription factor, FcrNAC22 is specifically induced in RL-irradiated fruits. FcrNAC22 localizes to the nucleus, and its gene expression is up-regulated as fruits change colour. Results from dual luciferase, yeast one-hybrid (Y1H) assays and electrophoretic mobility shift assays (EMSAs) indicate that FcrNAC22 directly binds to and activates the promoters of three genes (i.e. FcrLCYB1, FcrBCH2 and FcrNCED5) encoding key enzymes in carotenoid metabolic pathway. Moreover, FcrNAC22 overexpression in citrus and tomato fruits as well as in citrus callus enhances expression of a dozen of carotenoid biosynthetic genes including the aforementioned three, accelerates plastid conversion into chromoplasts, and promotes colour change. Knock down of FcrNAC22 expression in transient transformed citrus fruits attenuates fruit coloration induced by red light. Taken together, our results demonstrate that FcrNAC22 is an important transcription factor that mediates RL-induced fruit colouration via upregulation of carotenoid metabolism in citrus and perhaps in other plants.
\end{abstract}

\section{Hosted file}

manuscript.pdf available at https://authorea.com/users/373028/articles/490827-red-lightinduced-citrus-fruit-colouration-is-attributable-to-increased-carotenoid-metabolismregulated-by-the-nac-transcription-factor-fcrnac22

\section{Hosted file}

Highlights.pdf available at https://authorea.com/users/373028/articles/490827-red-lightinduced-citrus-fruit-colouration-is-attributable-to-increased-carotenoid-metabolismregulated-by-the-nac-transcription-factor-fcrnac22 

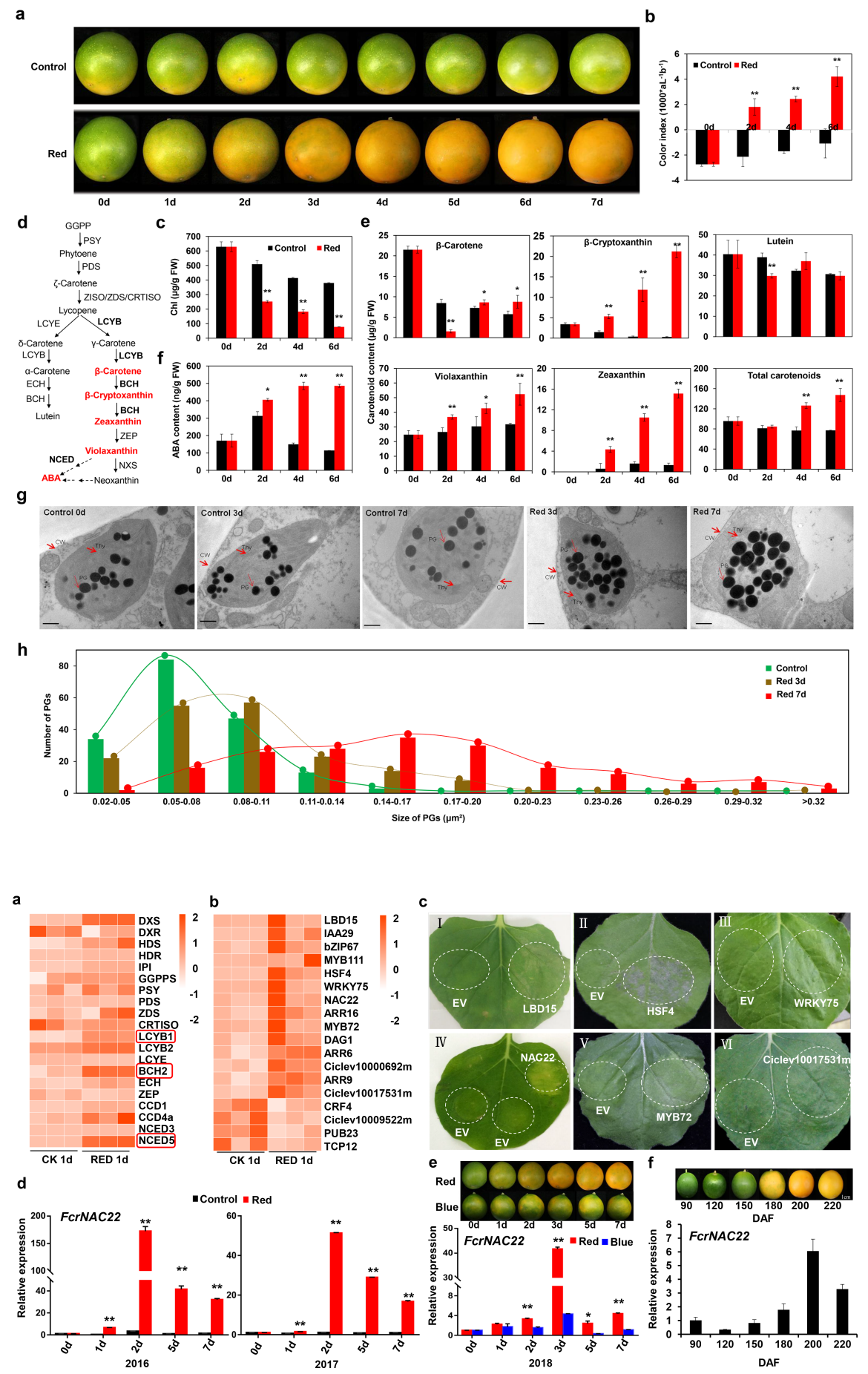

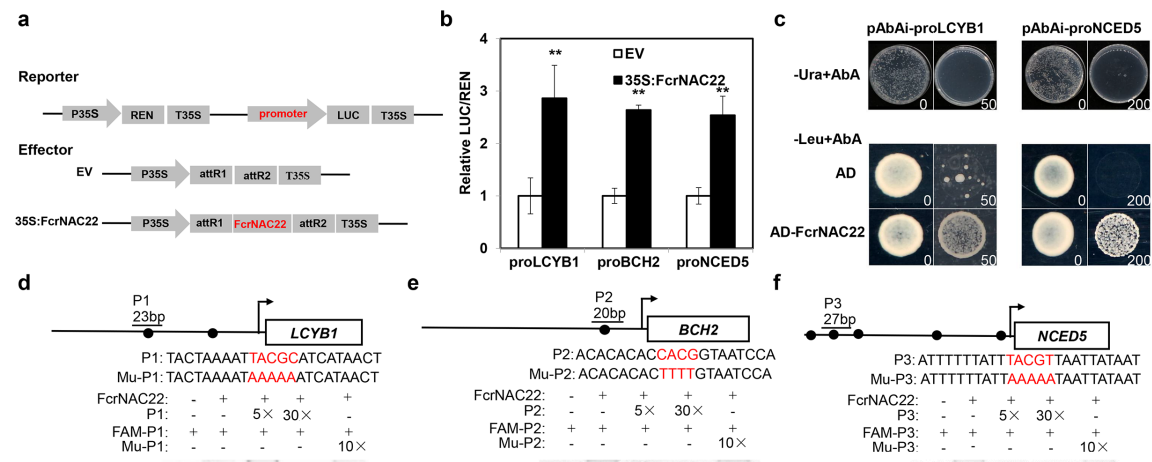

Shift band -

Shift band $\rightarrow$

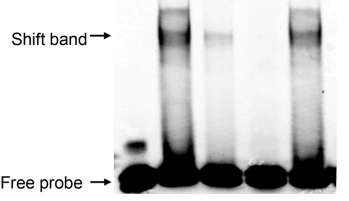

a
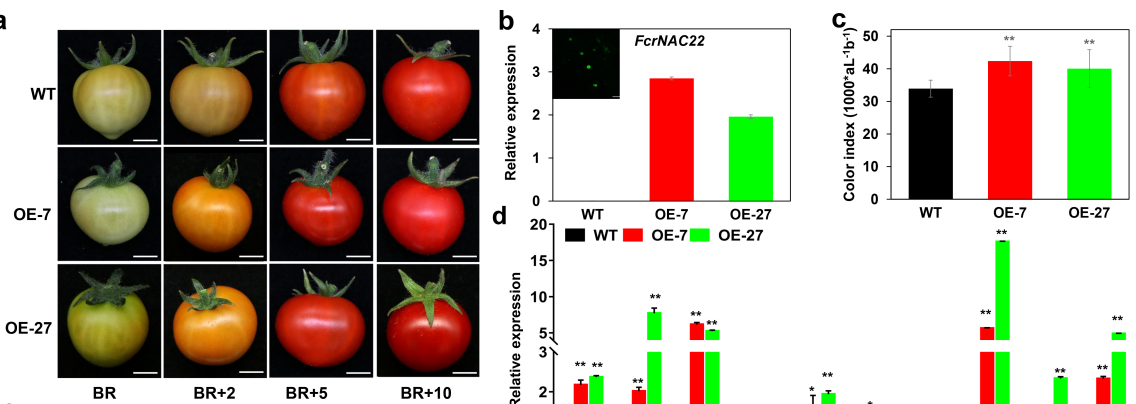

BR+10

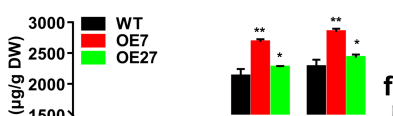

vivis

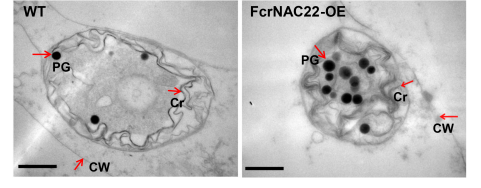

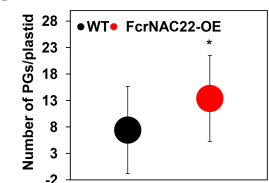



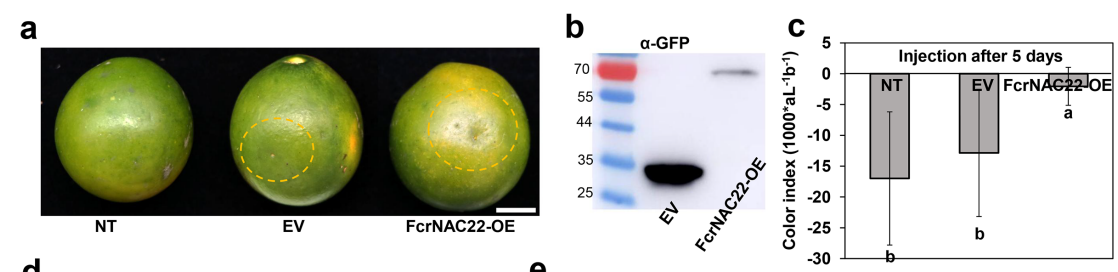

d

$\mathrm{e}_{100}$
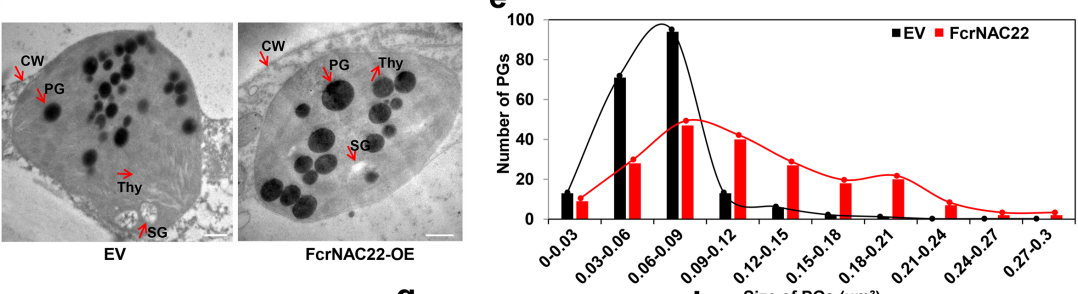

f
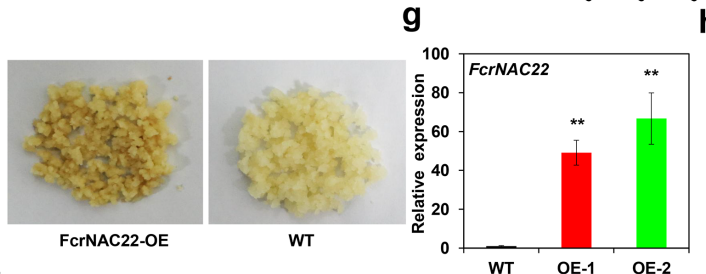

$h$ Size of PGs $\left(\mu \mathrm{m}^{2}\right)$
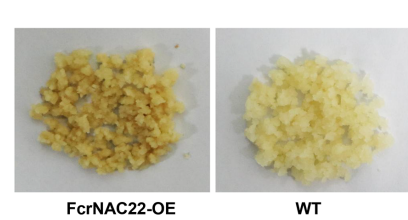

i
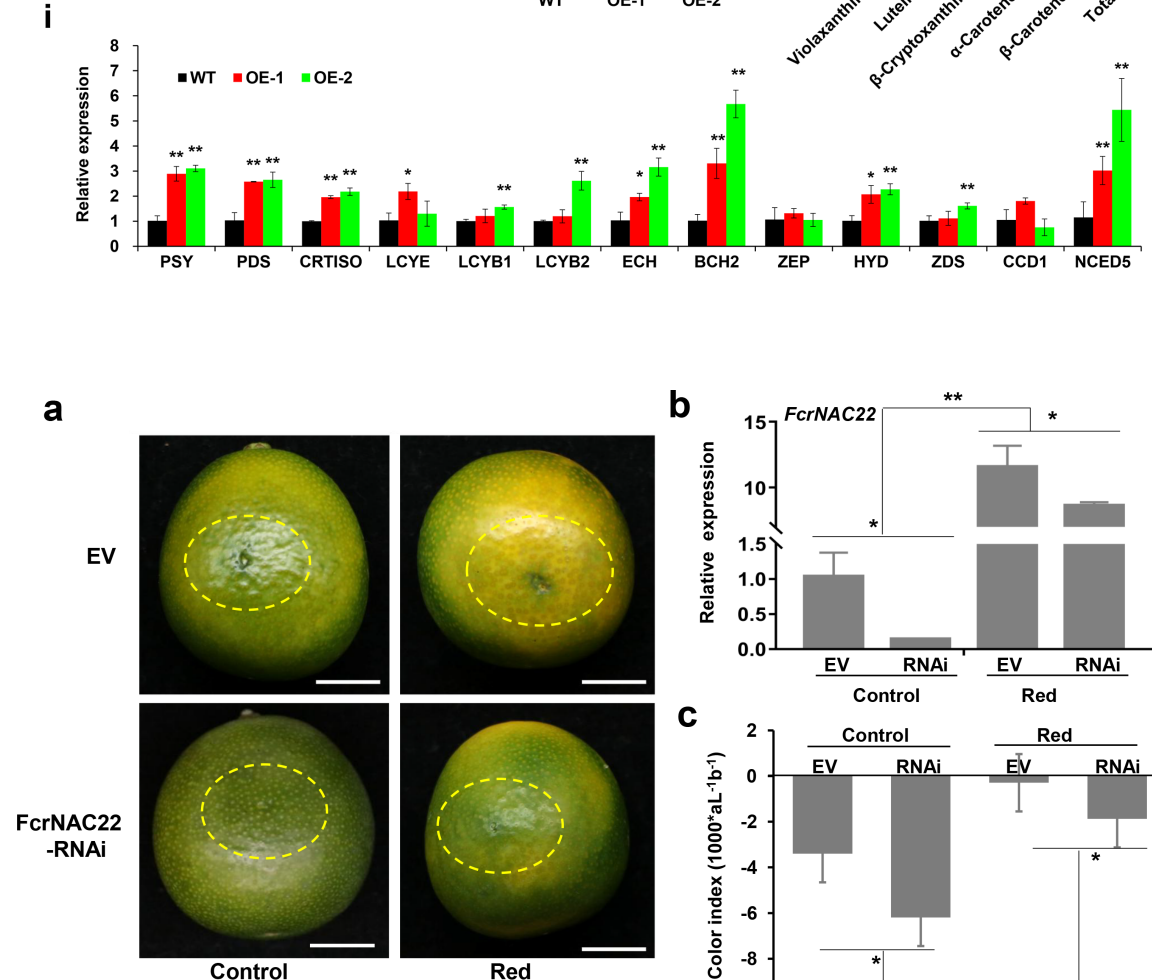

C
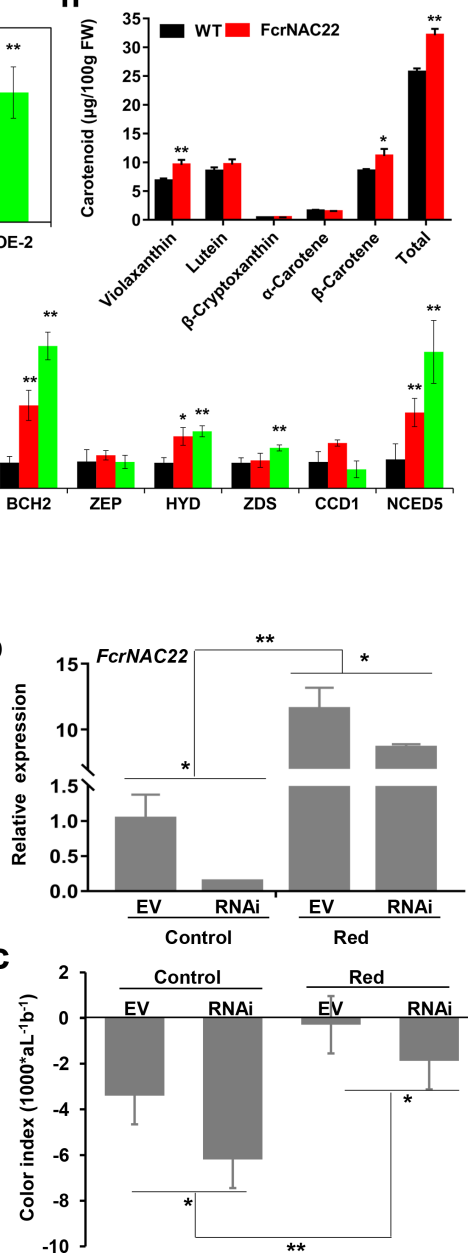


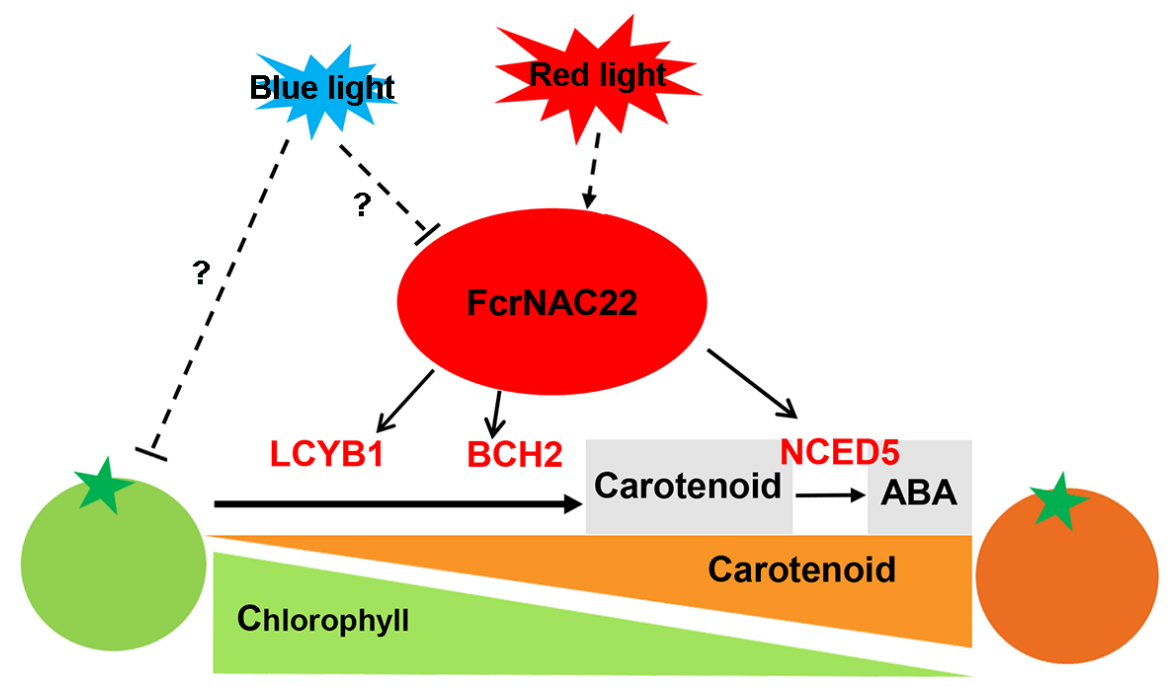

5 